\title{
A multicenter prospective phase III clinical randomized study of simultaneous integrated boost intensity-modulated radiotherapy with or without concurrent chemotherapy in patients with esophageal cancer: 3JECROG P-02 study protocol
}

Lin-rui Gao ${ }^{1}$, Xin Wang ${ }^{1}$, Weiming Han ${ }^{1}$, Wei Deng ${ }^{1}$, Chen Li', Xiaomin Wang ${ }^{2}$, Yidian Zhao ${ }^{2}$, Wenjie $\mathrm{Ni}^{1}$, Xiao Chang ${ }^{1}$, Zongmei Zhou' ${ }^{1}$, Lei Deng ${ }^{1}$, Wenqing Wang ${ }^{1}$, Wenyang Liu' ${ }^{1}$, Jun Liang ${ }^{1}$, Tao Zhang ${ }^{1}$, Nan Bi ${ }^{1}$, Jianyang Wang ${ }^{1}$, Yirui Zhai ${ }^{1}$, Qinfu Feng ${ }^{1}$, Jima LV ${ }^{1}$, Ling Li $^{3^{*}}$ and Zefen Xiao ${ }^{1 *}$ (i)

\begin{abstract}
Background: Since the development of three-dimensional conformal radiotherapy and intensity-modulated radiotherapy (IMRT), no prospective study has investigated whether concurrent chemoradiotherapy (SIB-IMRT with $60 \mathrm{~Gy}$ ) remains superior to radiotherapy (SIB-IMRT) alone for unresectable esophageal cancer (EC). Furthermore, the optimal therapeutic regimen for patients who cannot tolerate concurrent chemoradiotherapy is unclear. We recently completed a phase I/II radiation dose-escalation trial using simultaneous integrated boost (SIB), elective nodal irradiation, and concurrent chemotherapy for unresectable EC. We now intend to conduct a prospective, phase III, randomized study of SIB-IMRT with or without concurrent chemotherapy. We aim to find a safe, practical, and effective therapeutic regimen to replace the conventional segmentation (1.8$2.0 \mathrm{~Gy}$ ) treatment mode (radiotherapy \pm chemotherapy) for unresectable EC.

(Continued on next page)
\end{abstract}

\footnotetext{
*Correspondence: lilingtz@163.com; xiaozefen@sina.com

${ }^{3}$ Department of Oncology, Affiliated Tengzhou Central People's Hospital of Jining Medical University, Jining Medical University, Tengzhou 277599, China 'Department of Radiation Oncology, National Cancer Center/National Clinical Research Center for Cancer/Cancer Hospital, Chinese Academy of Medical Sciences and Peking Union Medical College, Beijing 100021, China Full list of author information is available at the end of the article
}

C C The Author(s). 2020 Open Access This article is licensed under a Creative Commons Attribution 4.0 International License, which permits use, sharing, adaptation, distribution and reproduction in any medium or format, as long as you give appropriate credit to the original author(s) and the source, provide a link to the Creative Commons licence, and indicate if changes were made. The images or other third party material in this article are included in the article's Creative Commons licence, unless indicated otherwise in a credit line to the material. If material is not included in the article's Creative Commons licence and your intended use is not permitted by statutory regulation or exceeds the permitted use, you will need to obtain permission directly from the copyright holder. To view a copy of this licence, visit http://creativecommons.org/licenses/by/4.0/ The Creative Commons Public Domain Dedication waiver (http://creativecommons.org/publicdomain/zero/1.0/) applies to the data made available in this article, unless otherwise stated in a credit line to the data. 


\begin{abstract}
(Continued from previous page)
Methods: This two-arm, open, randomized, multicenter, phase III trial will recruit esophageal squamous cell carcinoma patients (stage IIA-IVB [UICC 2002]; IVB only with metastasis to the supraclavicular or celiac lymph nodes). In all, 164 patients will be randomized using a 1:1 allocation ratio, and stratified by study site and disease stage, especially the extent of lymph node metastasis. Patients in the SIB arm will receive definitive SIB radiotherapy (95\% planning target volume/planning gross tumor volume, 50.4 Gy/59.92 Gy/28 f, equivalent dose in 2-Gy fractions = 60.62 Gy). Patients in the SIB + concurrent chemotherapy arm will receive definitive SIB radiotherapy with weekly paclitaxel and a platinum-based drug (5-6 weeks). Four cycles of consolidated chemoradiotherapy will also be recommended. The primary objective is to compare the 1-year, 2-year, and 3-year overall survival of the SIB + chemotherapy group and SIB groups. Secondary objectives include progression-free survival, local recurrence-free rate, completion rate, and adverse events. Detailed radiotherapy protocol and quality-assurance procedures have been incorporated into this trial.
\end{abstract}

Discussion: In unresectable, locally advanced EC, a safe and effective total radiotherapy dose and reasonable segmentation doses are required for the clinical application of SIB-IMRT + two-drug chemotherapy. Whether this protocol will replace the standard treatment regimen will be prospectively investigated. The effects of SIB-IMRT in patients with poor physical condition who cannot tolerate definitive chemoradiotherapy will also be investigated.

Trial registration: clinicaltrials.gov (NCT03308552, November 1, 2017).

Keywords: Esophageal cancer, Concurrent chemoradiotherapy, Definitive chemoradiotherapy/radiotherapy, Consolidated chemotherapy, Simultaneous integrated boost, Intensity-modulated radiotherapy, Randomized controlled trial

\section{Background}

The 2018 GLOBOCAN data estimated that approximately 572,000 people were newly diagnosed with EC in 2018, and that almost 509,000 people died of these cancers in the same year, making EC the seventh most common cancer and the sixth most common cause of cancer-related deaths [1]. In China, EC and esophagogastric junction cancer (EGJC) are the fouth most common types of cancer [2]; these malignancies always have a poor prognosis and respond poorly to treatment.

For patients with unresectable ECs (including patients with locally advanced EC or EGJC as well as patients who cannot undergo or refuse surgery), concurrent chemoradiotherapy is the standard treatment, and the recommended radiotherapy dose is 50.4 Gy based on the Radiation Therapy Oncology Group (RTOG) 85-01 [3, 4] and RTOG 94-05 trials [5]. However, these treatment and dose recommendations are currently considered controversial because of the following reasons. First, the randomized controlled trial part of the RTOG 85-01 study found that the 5-year overall survival (OS) rate after combined chemoradiotherapy was $26 \%$ compared with $0 \%$ following two-dimensional radiotherapy (2DRT) alone, which differs from the data reported in China [6, 7]. Over the past few decades, the 5-year OS rates after 2DRT with doses of 6070 Gy have been reported to vary from 8.4 to $14.6 \%$ [6-8]. Second, the follow-up evaluation of the RTOG 85-01 study showed that disease persistence and locoregional recurrence were common modes of treatment failure, especially in the primary tumor region [4]. While it was lower in group who received combined therapy. Therefore, increasing the local radiotherapy dose to the primary tumor might be required to improve local control [9]. However, as reported in the RTOG 94-05 study, patients receiving high-dose radiotherapy (64.8 Gy) showed no improvement in terms of OS or local control, as compared with patients receiving low-dose radiotherapy (50.4 Gy). Thus, the optimal radiation dose remains to be determined. Finally, three-dimensional conformal radiotherapy (3DCRT) for unresectable EC yields 5-year OS rates of 34-45.6\% [10-13], which is an improvement over the rates reported in the RTOG 85-01 and 94-05 studies. Moreover, radiotherapy (median dose, 60 Gy) with or without concurrent chemotherapy yields 5-year OS rates of 34.7 and $27.7 \%$, respectively [14]. These results do not show a large difference in 5-year OS between radiotherapy with concurrent chemotherapy and radiotherapy alone, unlike the findings reported in the RTOG $85-01$ study $(27.7 \%$ vs. $0 \%$, respectively). Although it was a retrospective study, it can also indicate that radiotherapy is the mainstay of treatment for EC, especially for patients who cannot tolerate concurrent chemotherapy. However, no prospective research study has been conducted to identify reasonable and effective doses of radiotherapy for EC.

The incidence of lymph node metastasis in EC is high, and the rate of early lymph node metastasis (i.e., in stage T1b) is $16.6-22.5 \%$ [15-17]; thus, preventive radiotherapy to the lymph nodes is essential. The simultaneous integrated boost (SIB) technique provides a suitable and heterogeneous dose distribution over a single radiation field. This technique is generally used to administer a high dose of irradiation to the tumor without significantly increasing the irradiation exposure of the organs at risk (OAR). However, as the esophagus has a lumen, administering a reasonable total dose of radiotherapy in multiple fractions is the basis of therapy. To evaluate this topic, we recently completed a 
phase I/II study of SIB intensity-modulated radiotherapy (IMRT) + two-drug chemotherapy for EC. We now intend to conduct a prospective, multicenter phase III clinical trial to determine whether SIB-IMRT with concurrent chemotherapy is sufficiently safe and effective to replace the standard treatment mode of conventional segmented radiotherapy $(1.8-2.0 \mathrm{~Gy})$ and concurrent chemotherapy. This study additionally aims to determine if SIB-IMRT alone is a suitable secondary treatment option for EC patients who cannot tolerate chemotherapy.

\section{Methods}

\section{Study design and objectives}

This study is an open label, randomized, comparative, multicenter study. The SIB technique will be used in this study, with the following dose regimen: 50.4 Gy/1.8 Gy/
$28 \mathrm{f}$ to the planning target volume (PTV) and $59.92 \mathrm{~Gy} /$ $2.14 \mathrm{~Gy} / 28 \mathrm{f}$ to the planning gross tumor volume (PGTV). Paclitaxel + nedaplatin will both be administered concurrent with radiotherapy. We randomly assigned (1:1) eligible patients, stratified by disease stage and tumor site, to one of four treatment groups: SIB + concurrent chemotherapy group or the SIB alone group. A flow chart giving an overview of the study design is shown in Fig. 1.

The coprimary objectives of this trial is to compare the 1year, 2-year, and 3-year OS rates of the SIB + chemotherapy group and the SIB alone group. The secondary objectives consist of similar comparisons of the progression-free survival rate, local recurrence-free survival rate, treatment completion rate, and rate of adverse events. Patient recruitment for this study was started on September 1, 2017, and the duration of enrollment will be approximately 5 years.

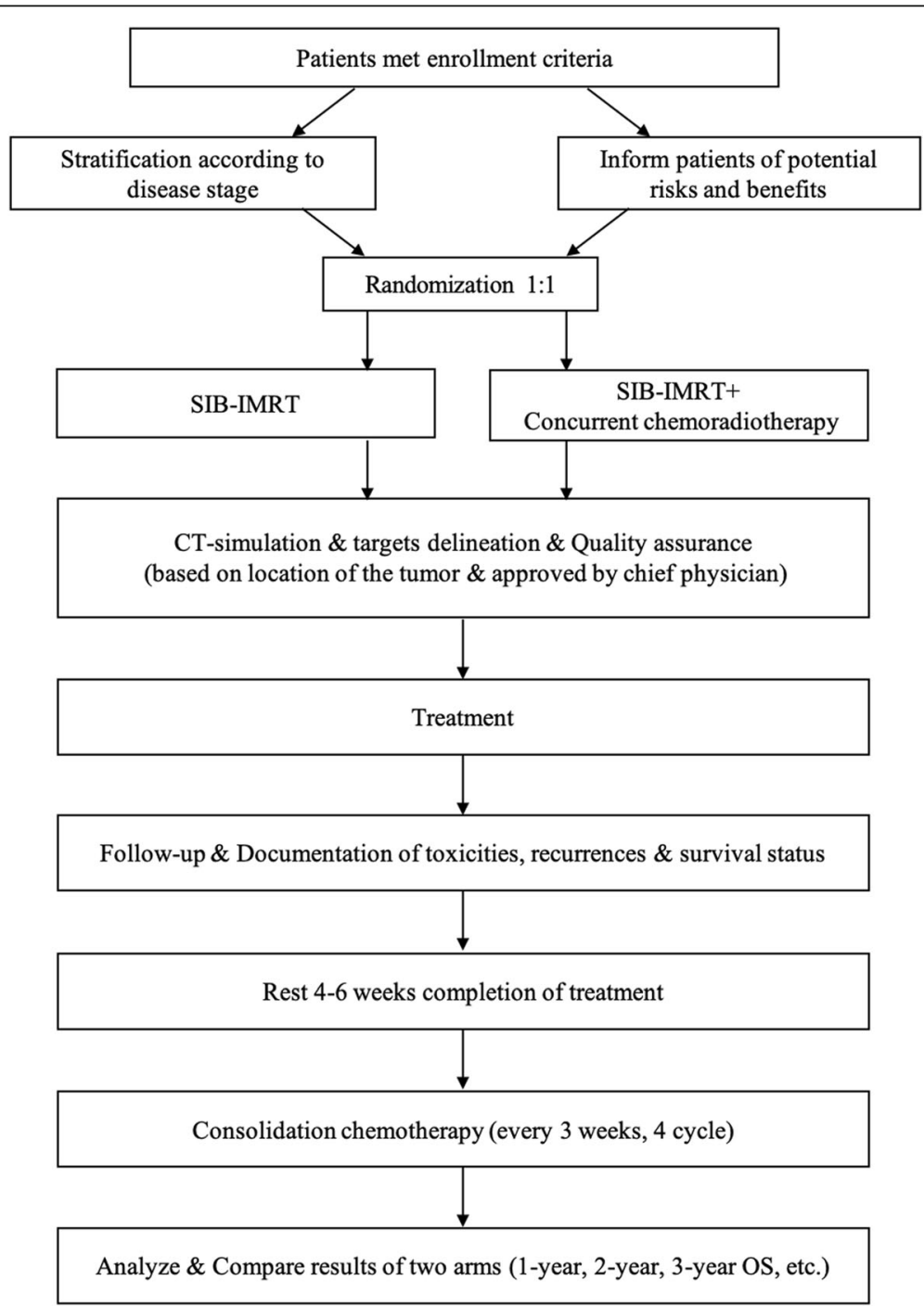

Fig. 1 Flow chart of the 3JECROG P-02 trial 


\section{Patient selection}

In this randomized phase III study, we recruited patients aged less than 70 years with histocytologically proven stage T2-4 N0-1 M1a (UICC 2002 [18]; stage IVB only with metastasis to the supraclavicular or celiac lymph nodes) unresectable esophagus squamous cell carcinoma (ESCC) of the are eligible for recruitment, no previous treatment before enrollment. Laboratory investigation requirements included the following: leukocytes $\geq 4.0 \times$ $10^{9} / \mathrm{L}$, neutrophils $\geq 3.5 \times 10^{9} / \mathrm{L}$, granulocytes $\geq 1.5 \times 10^{9} / \mathrm{L}$, platelets $\geq 100 \times 10^{9} / \mathrm{L}$, blood urea nitrogen $\leq 1.0 \times$ upper normal limit (UNL), creatinine $\leq 1.0 \times \mathrm{UNL}$, alanine aminotransferase/aspartate aminotransferase $\leq 1.5 \times \mathrm{UNL}$, alkaline phosphatase $\leq 1.5 \times \mathrm{UNL}$, and total bilirubin $\leq \mathrm{UNL}$. The general condition of the enrolled patients must also be acceptable: Karnofsky performance status score $\geq 70$ or Eastern Cooperative Oncology Group performance status score $\leq 1$, and Charlson Comorbidity Index score $\leq 3$.

The exclusion criteria include age $\geq 70$ years or $<18$ years, prior chemotherapy or radiotherapy, pregnancy or lactation, known drug allergy, refusal to provide informed consent, insufficient hepatorenal function, abnormalities on routine blood examination (as defined above), severe cardiovascular diseases, diabetes with uncontrolled blood sugar level, mental disorders, uncontrolled severe infection, and active ulceration requiring intervention.

The elimination criteria include the following: (1) assigned patients did not match the study requirements, and (2) patients whose treatment was not performed as planned, those who developed unacceptable toxicity reactions, or those who withdrew from the study on their own accord. The study termination criteria are as follows: (1) disease progression during treatment, (2) other diseases that significantly affect the general condition of the patients and necessitate cessation of treatment, (3) unacceptable treatment toxicity, and (4) voluntary withdrawal from the trial at any time, according to the patient's wishes.

\section{Radiotherapy}

After completing the pretreatment examination, the following procedures will be performed: enhanced computed tomography (CT) for positioning and outlining the target area, determining the dose to be prescribed according to the modified radiotherapy plan, and submitting it to the physician to formulate the radiotherapy plan. Once the chief physician approves the plan, radiotherapy can be started. Cone beam CT-guided radiotherapy will be performed at least three times in the first week of radiotherapy and once a week thereafter.

The gross tumor volume (GTV-T) is defined as the encompasses the primary tumor, and is determined using all available resources \{physical examination, upper gastrointestinal contrast, endoscopy, endoscopic ultrasonography [EUS], neck/thoracic/upper abdominal enhanced CT/MRI, positron-emission tomography [PET]CT (if necessary), etc.\}.

Lymph nodes diagnosed as metastatic or highly suspected as metastatic depending on the use of the physical examination and imaging tests (ultrasonography, CT, PET-CT, EUS, etc.) define as the metastatic regional nodes (GTV-N).

According to the clinical stage of the primary tumor and metastatic lymph nodes, the contouring of the clinical target volume (CTV) will be divided into two parts: elective nodal irradiation (ENI) and involved-field irradiation (IFI). ENI will include prophylactic irradiation of the draining lymph nodes. In such cases, the CTV is defined as the GTV with a $3.0-5.0 \mathrm{~cm}$ craniocaudal margin, a $0.6-0.8 \mathrm{~cm}$ lateral margin, and the corresponding draining lymph node area. For ECs with extensive lymphatic metastasis, beyond $5 \mathrm{~cm}$ of the primary tumor and multi-station lymph node metastasis, we will adopt IFI. The GTV with a $3.0-5.0 \mathrm{~cm}$ craniocaudal margin, a $0.6-0.8 \mathrm{~cm}$ lateral margin, and the GTV-N with a $1.0-$ $1.5 \mathrm{~cm}$ margin, including the metastatic lymph nodes together make up the CTV (Figs. 2 and 3).

The PGTV will be $1.0 \mathrm{~cm}$ craniocaudally beyond the GTV-T and $0.5 \mathrm{~cm}$ radially and the GTV-N. Planning target volume (PTV) will be defined as $0.5 \mathrm{~cm}$ margin of the CTV for tumor motion and set-up variations. The typical contouring of the targeted tumors in different locations is depicted in Figs. 2 and 3.

SIB-IMRT will be given 5 days per week (i.e., Monday to Friday with the weekend off) for an average of 5.5 weeks. Radiotherapy will be delivered to achieve a prophylactic dosage of $50.4 \mathrm{~Gy}(1.8 \mathrm{~Gy})$ to the PTV and 59.92 Gy $(2.14 \mathrm{~Gy})$ to the PGTV in 28 fractions. The contouring of the simulation images should include the lungs, heart, spinal cord, spinal cord planning OAR volume, and stomach on the CT scan. OARs such as the lungs, heart, spinal cord, and stomach will be delineated from their upper borders to their lower ends. The volume of lung tissue receiving $20 \mathrm{~Gy}$ or more should not exceed $28 \%$ of the total lung volume (i.e., V20<28\%) and the V30 should not exceed $20 \%$. The mean dose of the lung tissue should not be higher than $17 \mathrm{~Gy}$ (i.e., Dmean lung $\leq 17 \mathrm{~Gy})$. Other dose constraints to the OARs include the following: V40 heart < 30\%, V40 stomach $<40 \%$, Dmean spinal cord $=9-21 \mathrm{~Gy}$, and Dmax $\leq 45 \mathrm{~Gy} / 6$ weeks.

\section{Chemotherapy}

The concurrent chemotherapy regimen consists of weekly doses of paclitaxel and a platinum-based drug. Paclitaxel will be given at a dose of $45-60 \mathrm{mg} / \mathrm{m}^{2}$, once a week, concurrent with radiotherapy for 5-6 weeks. 


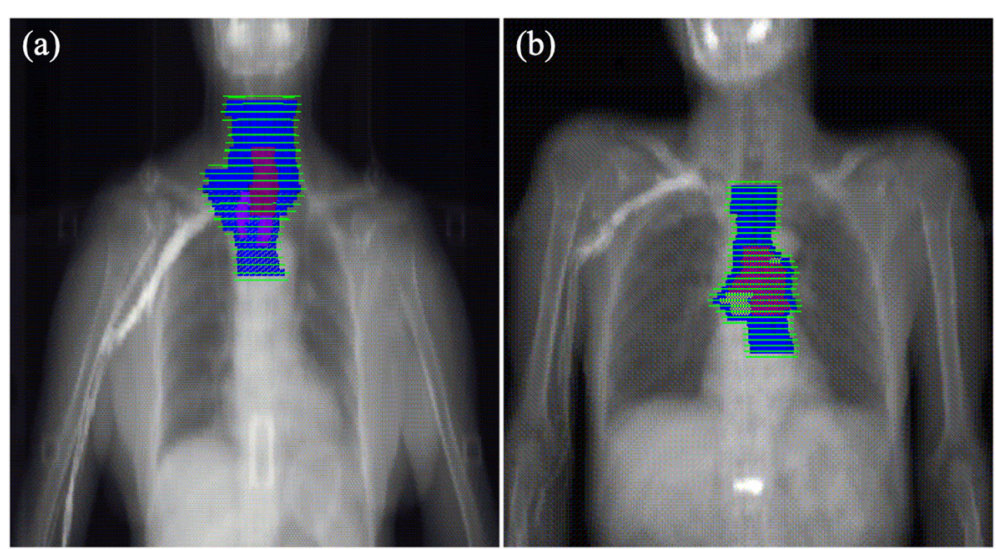

Fig. 2 Target contouring of (a) the cervical esophagus and (b) the middle thoracic esophagus (Mt). The red area indicates the gross tumor volume (GTV-T); the grey area, the gross tumor volume for lymph nodes (GTV-N); the blue area, the planning gross tumor volume (PGTV); and the green area, the planning target volume (PTV)

The dose of the platinum-based drug (nedaplatin, lobaplatin, or cisplatin) is $20-25 \mathrm{mg} / \mathrm{m}^{2}$, once a week, concurrent with radiotherapy for 5-6 weeks. A total of 5-6 cycles of concurrent chemotherapy are recommended depending on the patients' tolerance.
Consolidation chemotherapy within 1-3 months after the end of treatment will be recommended to appropriate and eligible patients who satisfy the following requirements: (1) Karnofsky performance status score $\geq 70$ points, (2) ability to have semi-liquid or solid foods or
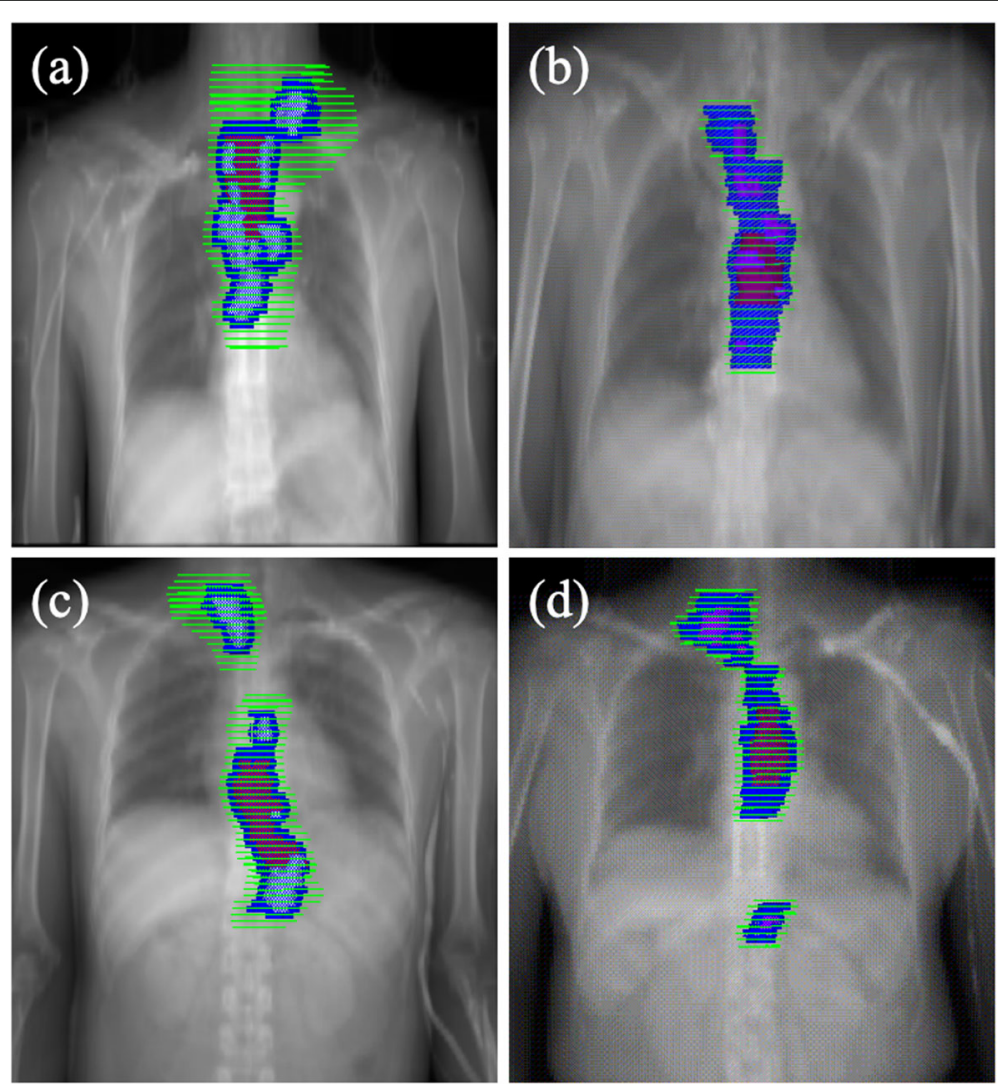

Fig. 3 Target contouring for $(\mathbf{a}, \mathbf{b})$ elective nodal irradiation (ENI) and ( $\mathbf{c}, \mathbf{d})$ involved-field irradiation (IFI). The red area indicates the gross tumor volume (GTV-T); the grey area, the gross tumor volume for lymph nodes (GTV-N); the blue area, the planning gross tumor volume (PGTV); and the green area, the planning target volume (PTV) 
receive nasal feeding, (3) no weight loss or loss of $<5 \%$ of the body weight, and (4) consent to undergo consolidation chemotherapy. The dose regimen for consolidation chemotherapy is as follows: paclitaxel $135-175 \mathrm{mg} /$ $\mathrm{m}^{2}$ on day 1 and a platinum-based drug (nedaplatin, lobaplatin, or cisplatin) $50-80 \mathrm{mg} / \mathrm{m}^{2}$ on days $1-2$ (lobaplatin $50 \mathrm{mg}$ on day 1 ) every 3 weeks for $2-4$ cycles starting 1-3 months after the completion of radiotherapy. Routine blood tests should be monitored every week, and hepatic and renal function should be checked during every chemotherapy cycle.

\section{Toxicity and adverse events}

All treatment-related toxicities and adverse events will be graded with the RTOG toxicity criteria and the Common Terminology Criteria of Adverse Events (version 4.0). The detailed adverse events will be recorded in patients' case report forms. Serious adverse events should be dealt with properly and reported to the institutional ethical review committee in $24 \mathrm{~h}$, and the patients treated as promptly as possible. All patients with severe adverse reactions should be followed up until recovery.

Concurrent chemotherapy will be terminated, if $\geq$ grade 2 anemia, thrombocytopenia, or hepatic or renal dysfunction, $\geq$ grade 4 leukopenia/neutropenia, $\geq$ grade 3 radiation esophagitis, or other $\geq$ grade 3 non-hematological toxicities occur. If adverse events de-grade to grade $0-1$ within 1 week of drug withdrawal, the patient can re-take chemotherapy as the required dose; otherwise, chemotherapy should be terminated. If $\geq$ grade 3 radiation pneumonitis occurs, both radiotherapy and chemotherapy should be terminated. The suitability of consolidation chemotherapy should be re-assessed within 4-8 weeks after radiotherapy, regardless of the grade of toxicities developed during definitive chemoradiotherapy.

\section{Statistical analysis and sample-size considerations}

We assume that an estimated difference in 1-year OS of $33 \%$ (SIB arm) versus 50\% (SIB + concurrent chemotherapy arm) [19] would justify applying this regimen in the future. Assuming a one-sided significance level of 0.05, a power of 0.80 , and $10 \%$ of loss in each arm, a total of 164 patients ( $n=82$ in each group) would be needed in this trial. After using SAS software to generate a random number table, the patients will be randomly divided into two groups.

The rates of OS will be estimated using the KaplanMeier method, and the distributions of OS will be compared using the log-rank tests. Cox regression analysis will be used to identify prognostic factors for survival benefit.

\section{Ethics}

The enrolled patients should be informed of the background of both treatment options, especially known efficiency and toxicities by the doctor-in-charge. It must be emphasized that both before and during the study, the patient is allowed to refuse the treatment. Before enrollment, the patients should sign literal informed consent. This study will be carried out accordance with the "Declaration of Helsinki" or the laws and regulations of the country under the supervision of the principal investigator, in order to provide the individual with greater protection. The institutional ethical review committee has approved with this study.

\section{Follow-up}

Tumor regression should be assessed per the Response Evaluation Criteria in Solid Tumors (RECIST, version 1.1) within 1-2 months after the completion of treatment. The therapeutic effect on measurable metastatic lymph nodes and primary esophageal tumors will be evaluated using upper gastrointestinal contrast, endoscopy, EUS, neck/thoracic/upper abdominal enhanced CT/MRI, PET-CT (if necessary), etc.

The follow-up assessments will be done every 3 months for the first 2 year, every 6 months for $3-5$ years, then every year. Routine follow-up assessments included: (a).

assessing tumor-related symptoms of dysphagia, chest tightness, hoarseness, cough, fever, etc., (b) laboratory investigations of blood routine examination, hepatic and renal function, tumor markers, etc., (c) image examinations of contrast-enhanced CT of the neck, thorax, and abdomen, ultrasonography of the neck and abdomen, upper gastrointestinal contrast, bone scan (if bone pain or abnormally elevated alkaline phosphatase), MRI of the brain (in case of any symptoms related to the central nervous system), etc., (d) recording of the patients' vital signs, performance status, disease progression, subsequent treatment, nutrition, life quality, and any adverse events, etc.

\section{Quality assurance}

A strict coordination and monitoring system will be constructed for this trial. First, a consist of physicians, dosimetrists, medical physicians, and research fellows' team, named as Radiotherapy Trials Quality Assurance (RTTQ A), has been created before the start of enrollment. A censor in charge of the RTTQA team will evaluate and audit the quality of data collected, communicate with the physicians from all participating centers.

In the assurance of treatment equality and quality of all involved centers, we have made great effort. We selected an EC case, an example by the RTTQA team, sent the case and CT imaging data to all participating centers at the start of the study. Then, all participating centers were requested to send the target delineation back to the RTTQA team. The RTTQA team assessed all collected cases for major and minor deviations. This is the 
first round of collection of target delineation (CTD). After that, a detailed protocol for target delineation was sent to the all centers and the physicians in charge contoured the targets again on the same sample case [20] and sent back again (second round of CTD). The RTTQA team examined the radiotherapy plans thoroughly and found both the quality and equality of the plans had improved significantly after two rounds of CTD. This procedure ensures that all centers and investigators have had the abilities and qualifications of planned test case before recruiting the patients. During the study, the censors from the RTTQA team will inspect randomly the quality of treatment, including images, target delineation, radiotherapy plans, and doses.

\section{Discussion}

For unresectable EC, the National Comprehensive Cancer Network (NCCN) [21] and European Society for Medical Oncology (ESMO) [22] recommend a dose of 50-50.4 Gy for definitive radiotherapy with concurrent dual-drug intravenous chemotherapy (fluorouracil/capecitabine + a platinum-based drug), based on the RTOG 85-01 [3] and RTOG 94-05 studies [5]. However, these recommendations are based on 2DRT in the 1990s. The main cause of failure of this treatment is the high rate of locoregional recurrence ( $\geq 50 \%)$; moreover, treatment with a higher dose of 61-65 Gy with concurrent chemotherapy does not improve treatment outcomes as compared with the same regimen with a dose of $50 \mathrm{~Gy}$ [23]. Therefore, a reasonable radiotherapy dose supported by more research data is required. In the past several decades, few prospective studies have been conducted on the dose of 3DCRT, including SIB-IMRT, with concurrent chemotherapy. Retrospective analyses in our center show that for unresectable ECs, the 5-year OS (22.1$27.7 \%$ ) after 3DCRT alone (median dose, $60 \mathrm{~Gy}$ ) [14, 24] is higher than that after 2DRT (8.3-14.3\%) [25]. These data confirm that the application of 3DCRT has improved the survival rate, and 3DCRT is now the main treatment for EC. Advancements in imaging technology have made radiotherapy more accurate, which may have improved its curative effects. Considering that radiotherapy (dose, > 50 Gy) with concurrent chemotherapy has been reported to yield 5-year OS rates of $26.0-44.3 \%$, this treatment strategy is now the preferred option for EC [19, 26-28]. Compared with radiotherapy alone, radiotherapy with concurrent chemotherapy improves the 5 -year OS rate by $2-11.1 \%[27,29]$, which is different from the survival gap reported in RTOG85-01 (26\% vs. $0 \%$ ) [8]. Furthermore, 3DCRT is an effective treatment, second only to definitive chemoradiotherapy, especially for patients who cannot tolerate chemotherapy. Therefore, a prospective research study on this treatment strategy is required.
Currently, there is no international consensus on whether the draining lymph nodes need preventive irradiation in EC. A large body of data on three-field lymph node dissection in Japanese patients with EC has provided detailed lymph node metastasis sites and rates, and lends clinical support to the use of preventive regional lymph node irradiation in patients with unresectable EC (i.e., radiation to the high-risk lymph node metastasis area) [30, 31]. However, the dose required for preventive lymph node irradiation is different from that required for the primary tumor site. In the era of conventional radiotherapy technology, we had to undertake fractional or sequential treatments to meet the different dose requirement. However, by using reverse intensity modulation feature of IMRT, different radiation dose distributions can be administered to the nodal area and the primary tumor site at the same time. A phase II study of radical IMRT combined with concurrent chemotherapy for EC was performed with a similar dose as that used in the high-dose group of the RTOG 94-05 study. The median survival time (MST) was 23 months, and the 3-year OS rate was $44.4 \%$, which indicates that SIB might be effective [32].

The use of SIB-IMRT is a novel aspect of our study. The long-term follow-up results of the RTOG 85-01 study showed that the major patterns of treatment failure were primary tumor persistence (radiotherapy: 37\% vs. chemoradiotherapy: 25\%) and locoregional failure (radiotherapy: $16 \%$ vs. chemoradiotherapy: $13 \%$ ), which indicates that the local control rate for doses under 50.4 Gy is not satisfactory [33]. Thus, higher doses may be necessary for primary tumor areas, without increasing the toxicity to the surrounding normal tissue. One retrospective study also found that among ESCC patients, those who received high-dose irradiation ( $\geq 60 \mathrm{~Gy}$ ) had better OS and local control rates than those who only received the conventional dose (50.4 Gy) [34]. Therefore, to explore this problem, we conducted a phase I/II radiation dose-escalation trial using the SIB technique with ENI and concurrent chemotherapy for unresectable EC [35]. We found that the SIB technique was feasible and safe at the maximum tolerated dose $[95 \% \mathrm{PGTV} / \mathrm{PTV}=$ 59.92 (equivalent dose in 2-Gy fractions or $\mathrm{EQD} 2=$ $60.62 \mathrm{~Gy}) / 50.40 \mathrm{~Gy} / 28 \mathrm{f}$ ] concurrent with ENI and dualdrug chemotherapy for patients with unresectable EC. A total of 53 patients with SCC were enrolled in the above study. The median OS time, 1-year OS rate, and 1-year local failure-free survival were 31 months, 76.9, and $78.8 \%$, respectively. Compared with a recent phase I/II trial of chemoradiotherapy with SIB radiotherapy for unresectable locally advanced EC (95\% PGTV/PTV = $63.00 \mathrm{~Gy} / 50.40 \mathrm{~Gy} / 28$ f, EQD2 = 64.31 Gy), our study had a better median OS, lower 1-year local recurrence rates, and similar 1-year OS and 1-year local recurrence 
rates (21.5 months, 30, and $78.3 \%$ respectively) [36]. However, all of these studies require long-term followup. Therefore, we intend to apply the above dose regimen in this phase III study to determine whether this regimen is safe, reliable, and promising.

The 5-year OS rate of EC patients has shown varying degrees of improvement after definitive radiotherapy with IMRT; even in the era of 2DRT, the 5-year OS was not $0 \%$. Certain EC patients, such as those who are elderly or frail, those in poor health, and those with complications, are considered ineligible for esophagectomy. In such patients, definitive radiotherapy without major toxicity is considered a promising alternative. The NCCN and ESMO recommended dual-drug intravenous chemotherapy regimen (fluorouracil/capecitabine $+\mathrm{a}$ platinum-based drug) may cause severe acute and late adverse effects and is related to poor compliance rates in this specific population. Thus, radiotherapy alone might provide lower toxicity, and better survival and quality of life for these patients, and might be the preferred choice of treatment.

The widely accepted SIB-IMRT fractionated dose and total dose for preventive nodal irradiation are $1.8 \mathrm{~Gy}$ and 50.4 Gy, respectively. In contrast, the SIB-IMRT dose for the primary treatment area is controversial. The fractionated dose varies from 1.8 to $2.8 \mathrm{~Gy}$; the total dose, from $62.5 \mathrm{~Gy}$ to $70 \mathrm{~Gy}$; and the number of fractions, from 25 to 36, which reflects a wide variation [36-38]. Moreover, in the RTOG 85-01 and RTOG 94-05 studies, a total radiation dose of 64 Gy did not show significant benefits. Thus, a study to determine the appropriate radiotherapy dose and dose stratification is critical. Tan et al. reported that propensity score matching of $480 \mathrm{pa}-$ tients with ESCC receiving definitive radiotherapy or chemoradiotherapy (radiation dose: 50-70Gy) showed that: in 60-70Gy radiation dose range, there was no difference in OS rate between the radiotherapy group and chemoradiotherapy group $(1,3$, and 5 years OS: 66.0, $35.6,25.6 \%$ vs $63.6,35.0,25.3 \%, p=0.833)$. While the OS rate after radiation and concurrent chemotherapy was significantly higher in the 50-59.9 Gy dose group (1, 3, and 5 years OS: 70.0, 36.4, and 32.3\%; MST: 20 mouths] than in the radiotherapy group $(1,3$, and 5 years OS: 57.1, 23.9, and 12.0\%; MST: 15 months; $p=0.030$ ) [24]. However, in the above study, the patients treated with this dose range (2.2-2.25 Gy/62.5-66 Gy/25-30 f) may be highly selected, for example, patients in whom the primary tumor was not sensitive to treatment, especially patients with EC who showed insignificant tumor regression during radiotherapy; or no signs of ulcer perforation without T4 stage. However, it is difficult to predict whether the tumor will be sensitive to radiotherapy before the treatment. Many studies on preoperative chemoradiotherapy/radiotherapy (neoadjuvant therapy) for
EC have reported pathological complete response rates of $29-54.1 \%$ [39-44], while the rates of partial response or no response account for a higher proportion of patients. Moreover, pathological response is significantly associated with disease recurrence and survival [42-44]. In our phase I/II study, one EC patient received 2.17-Gy fractionated doses and 28-fraction radiotherapy, and he developed esophageal perforation during treatment. Therefore, whether SIB-IMRT (2.2-2.25 Gy/62.5-66 Gy/ 25-30 f) can replace conventional radiotherapy (1.8-2.0 Gy/50-50.4 Gy) as the standard treatment needs to be determined using phase III studies. A retrospective analysis of $2762 \mathrm{EC}$ patients in China found that a total radiation dose of $60-61.9 \mathrm{~Gy}$ or $62-63.9 \mathrm{~Gy}$ in EQD2 produced the highest 5-year OS rates (31.7 and 34.7\%, respectively); however, the 5-year OS rate was only 23$27.4 \%$ in the $\geq 64$ Gy group [14]. Although survival is affected by various factors, this result indicates that more prospective studies are needed to find the reasonable dose. Establishing a reasonable total dose and fractionated dose is crucial for the clinical application of SIB-IMRT. However, there is no related evidence-based research to determine whether high-dose radiotherapy can yield better locoregional control and survival benefit for patients diagnosed with residual tumor during treatment.

Preventive regional irradiation and concurrent chemotherapy can improve the local control rate by eliminating micrometastases. However, whether these measures can increase the OS rate is not certain. It is reported that concurrent chemotherapy can increase the control of micrometastases, which might provide a possible survival benefit [33]. In the RTOG 85-01 study, the concurrent chemotherapy regimen consisted of cisplatin and fluorouracil. A 2012 randomized study of preoperative neoadjuvant chemoradiotherapy versus surgery alone for EC patients showed that the pathological complete response rate was $49 \%$ after weekly paclitaxel and carboplatin chemotherapy [45]. However, only 37 ESCC patients were recruited in this study. Thus, whether SIB-IMRT plus concurrent chemotherapy can be an alternative to conventional radiotherapy in ESCC patients' needs to be determined.

In this paper, we propose a prospective, multicenter phase III clinical trial to obtain high-level type I evidence for a safe and effective therapeutic regimen for patients with unresectable EC. We will compare SIB-IMRT with or without concurrent paclitaxel + nedaplatin chemotherapy with the addition of consolidation chemotherapy for advanced EC.

\section{Abbreviations}

IMRT: Intensity-Modulated Radiotherapy; EC: Esophageal Cancer;

SIB: Simultaneous Integrated Boost; EGJC: Esophagogastric Junction Cancer; RTOG: Radiation Therapy Oncology Group; OS: Overall Survival; 2DRT: TwoDimensional Radiotherapy; 3DCRT: Three-Dimensional Conformal 
Radiotherapy; OAR: Organs at Risk; PTV: Planning Target Volume; PGTV: Planning Gross Tumor Volume; UNL: Upper Normal Limit; CT: Computed Tomography; GTV-T: Gross Tumor Volume; EUS: Endoscopic Ultrasonography; PETCT: Positron-Emission Tomography; GTV-N: Metastatic Regional Nodes; CTV: Clinical Target Volume; ENI: Elective Nodal Irradiation; IFI: Involved-Field Irradiation; RECIST: Response Evaluation Criteria in Solid Tumors; RTTQA: Radiotherapy Trials Quality Assurance; CTD: collection of target delineation; NCCN: National Comprehensive Cancer Network; ESMO: European Society for Medical Oncology; MST: Median Survival Time; ESCC: Esophageal Squamous Cell Carcinoma; EQD2: Equivalent dose in 2-Gy fractions

\section{Acknowledgements}

We thank all the patients who participated in this trial, all participating branch-centers and investigators who devote their time and passion in the implementation of this study. We thank Jing-Jin-Ji Esophageal and Esophagogastric Cancer Radiotherapy Oncology Group (3JECROG) and Beijing branch of the Chinese Medical Association for the opportunity of initiating this prospective multi-center phase III trial. The following list of names show the investigators who contributed this study by making substantial contributions to the delivery of the study: Nan Bi, Qinfu Feng, Jima Lv, Tao Zhang, Wei Deng, Weiming Han, ect. Tian Yuan gave guiding opinions on the quality control of radiophysics and radiotherapy plans.

\section{Trial status}

The study protocol was approved by the institutional review board in July 2017. Recruitment started in September, 2017 and is currently ongoing.

\section{Authors' contributions}

ZFX and LL made substantial contributions to the conception and design of the study, revised the article critically for important intellectual content and gave final approval of the version to be published; XW made contributions to the design of the study, gave substantial contributions to the organization of this trial and revised the article critically; LRG draft the manuscript and ZFX revised the manuscript; YDZ participated in designing and conducting the study; WMH, WD, CL, XMW, WJN and XC made substantial contribution to the delivery of this study and collected data; $Z M Z, L D, W Q W, W Y L, J L, T Z$, NB, JYW, YRZ, QFF and JML are currently involved in study implementation. All authors read and approved the final manuscript.

\section{Funding}

Beijing Hope Run Special Fund of Cancer Foundation of China (LC2016L04). The funding source has no role in study design, data collection, analysis, interpretation, the writing of the manuscript, or the decision to submit the current study.

\section{Availability of data and materials}

Not applicable - data collection is still ongoing.

\section{Ethics approval and consent to participate}

Institutional review board approval was obtained for the 3JECROG P-02 trial from the ethical committee of the Chinese Academy of Medical Sciences (reference number 17-113/1369). The 3JECROG P-02 trial is published under NCT03308552 on ClinicalTrials.gov. Written informed consent is obtained from all participants.

\section{Consent for publication}

Not applicable.

\section{Competing interests}

The authors have declared that no competing interests exist.

\footnotetext{
Author details

'Department of Radiation Oncology, National Cancer Center/National Clinical Research Center for Cancer/Cancer Hospital, Chinese Academy of Medical Sciences and Peking Union Medical College, Beijing 100021, China. ${ }^{2}$ Department 4th of Radiation Oncology, Anyang Cancer Hospital, Anyang 455000, China. ${ }^{3}$ Department of Oncology, Affiliated Tengzhou Central People's Hospital of Jining Medical University, Jining Medical University, Tengzhou 277599, China.
}

Received: 22 July 2020 Accepted: 8 September 2020 Published online: 22 September 2020

\section{References}

1. Bray F, Ferlay J, Soerjomataram I, Siegel RL, Torre LA, Jemal A. Global cancer statistics 2018: GLOBOCAN estimates of incidence and mortality worldwide for 36 cancers in 185 countries. CA Cancer J Clin. 2018;68(6):394-424.

2. Chen WQ, Zheng RS, Baade PD, Zhang SW, Zeng HM, Bray F, Jemal A, Yu XQ, He J. Cancer statistics in China, 2015. CA Cancer J Clin. 2016;66(2):115-32.

3. Herskovic A, Martz K, Alsarraf M, Leichman L, Brindle J, Vaitkevicius V, Cooper J, Byhardt R, Davis L, Emami B. Combined chemotherapy and radiotherapy compared with radiotherapy alone in patients with Cancer of the esophagus. New Engl J Med. 1992;326(24):1593-8.

4. Cooper JS, Guo MD, Herskovic A, Macdonald JS, Martenson JA, Al-Sarraf M, Byhardt R, Russell AH, Beitler JJ, Spencer S, et al. Chemoradiotherapy of locally advanced esophageal cancer - long-term follow-up of a prospective randomized trial (RTOG 85-01). JAMA. 1999;281(17):1623-7.

5. Minsky BD, Pajak TF, Ginsberg RJ, Pisansky TM, Martenson J, Komaki R, Okawara G, Rosenthal SA, Kelsen DP. INT 0123 (radiation therapy oncology group 94-05) phase III trial of combined-modality therapy for esophageal cancer: high-dose versus standard-dose radiation therapy. J Clin Oncol. 2002;20(5):1167-74.

6. Department of Radiology SCH. A clinical analysis of 1034 cases of esophageal cancer treated by radiotherapy. Cancer Res Prev Treat. 1978;6(4): 46-51.

7. Yin W, Zhang L, Yang Z, Miao Y, Yu Z, Zhang Z, Zhuang G, Wang M, Li G, Fan $L$, et al. An analysis of 3,798 cases of esophageal cancer treated by radiation. Chinese J Radiat Oncol. 1980;2(3):216-21.

8. Cooper JS, Guo MD, Herskovic A, Macdonald JS, Martenson JA Jr, Al-Sarraf M, Byhardt R, Russell AH, Beitler JJ, Spencer S, et al. Chemoradiotherapy of locally advanced esophageal cancer: long-term follow-up of a prospective randomized trial (RTOG 85-01). Radiation Therapy Oncology Group. JAMA. 1999;281 (17):1623-7.

9. Kawaguchi Y, Nakamura S, Miyagi K, Nakajima A, Suzuki O, Nishiyama K. Patterns of failure in patients with clinical stage IA thoracic esophageal Cancer treated with definitive radiotherapy using localized field. Int I Radiat Oncol. 2010;78(3):S198-9.

10. Lin SH, Wang L, Myles B, Thall PF, Hofstetter WL, Swisher SG, Ajani JA, Cox $J D$, Komaki R, Liao Z. Propensity score-based comparison of long-term outcomes with 3-dimensional conformal radiotherapy vs intensitymodulated radiotherapy for esophageal cancer. Int J Radiat Oncol Biol Phys. 2012;84(5):1078-85.

11. Deng JY, Wang C, Shi XH, Jiang GL, Wang Y, Liu Y, Zhao KL. Reduced toxicity with three-dimensional conformal radiotherapy or intensitymodulated radiotherapy compared with conventional two-dimensional radiotherapy for esophageal squamous cell carcinoma: a secondary analysis of data from four prospective clinical. Dis Esophagus. 2016;29(8):1121-7.

12. Chen NB, Qiu B, Zhang J, Qiang MY, Zhu YJ, Wang B, Guo JY, Cai LZ, Huang SM, Liu MZ, et al. Intensity-modulated radiotherapy versus threedimensional conformal radiotherapy in definitive Chemoradiotherapy for cervical esophageal squamous cell carcinoma: comparison of survival outcomes and toxicities. Cancer Res Treat. 2020;52(1):31-40.

13. Xu D, Li G, Li H, Jia F. Comparison of IMRT versus 3D-CRT in the treatment of esophagus cancer. Medicine. 2017;96(31):e7685.

14. Wang X, Wang L, Chen J, Zhang W, Wang X, Ge X, Shen W, Hu M, Yuan Q, $X u Y$, et al. Prognostic analysis of definitive three-dimensional radiotherapy for non-surgically resectable esophageal squamous cell carcinoma:a multicenter retrospective study (3JECROG R-01). Chinese J Radiat Oncol. 2018; 27(11):959-64.

15. Duan XF, Tang P, Shang XB, Jiang HJ, Yu ZT. The prevalence of lymph node metastasis for pathological $\mathrm{T} 1$ esophageal cancer: a retrospective study of 143 cases. Surg Oncol. 2018;27(1):1-6.

16. Dubecz A, Kern M, Solymosi N, Schweigert M, Stein HJ. Predictors of lymph node metastasis in surgically resected T1 esophageal Cancer. Ann Thorac Surg. 2015;99(6):1879-85 discussion 1886.

17. Merkow RP, Bilimoria KY, Keswani RN, Chung J, Sherman KL, Knab LM, Posner $M C$, Bentrem DJ. Treatment trends, risk of lymph node metastasis, and outcomes for localized esophageal cancer. J Natl Cancer Inst. 2014;106(7): dju133.

18. Cancer AJCo. Sixth edition of the AJCC Cancer staging manual: Library of Congress Cataloging-in-Publication Data. Springer Verlag; 2002. 
19. Herskovic A, Martz K, Al-Sarraf M, Leichman L, Brindle J, Vaitkevicius V, Cooper J, Byhardt R, Davis L, Emami B. Combined chemotherapy and radiotherapy compared with radiotherapy alone in patients with cancer of the esophagus. N Engl J Med. 1992;326(24):1593-8.

20. Xiao Z, Zhou Z, Li Y. Esophageal Cancer target volume delineation and treatment guidance for radiation therapy (in Chinese). 1st ed. Beijing: People's Medical Publishing House; 2017.

21. Soce M, Bisof V, Rakusic Z, Krpan AM, Stancic-Rokotov D, Sandrk S, Juretic A. Treatment for esophageal and esophagogastric junction cancer with radical radiotherapy: a single-institution cohort study. Ann Oncol. 2019;30:e023190.

22. Lordick F, Mariette C, Haustermans K, Obermannova R, Arnold D, Comm EG. Oesophageal cancer: ESMO clinical practice guidelines for diagnosis, treatment and follow-up. Ann Oncol. 2016;27:v50-7.

23. Brower JV, Chen S, Bassetti MF, Yu M, Harari PM, Ritter MA, Baschnagel AM. Radiation dose escalation in esophageal Cancer revisited: a contemporary analysis of the National Cancer Data Base, 2004 to 2012. Int J Radiat Oncol Biol Phys. 2016;96(5):985-93.

24. Tan L, Xiao ZZ, H. , Chen D, Feng Q, Zhou Z, Lyu J, Liang J, Yin W: Survival comparison of three-dimensional radiotherapy alone with concurrent chemoradiotherapy for non-surgical carcinoma. Chinese J Radiat Oncol 2015, 24(2):106-110.

25. Xiao Z, Jiang J, Yin W. Radiotherapy for carcinoma of the esophagus: Progress of treatment and research in China. Chin J Clin Oncol. 2006;3(5): 305-14.

26. Hihara J, Hamai Y, Emi M, Murakami Y, Kenjo M, Nagata Y, Okada M. Role of definitive chemoradiotherapy using docetaxel and 5-fluorouracil in patients with unresectable locally advanced esophageal squamous cell carcinoma: a phase II study. Dis Esophagus. 2016;29(8):1115-20.

27. Kumar S, Dimri K, Khurana R, Rastogi N, Das KJ, Lal P. A randomised trial of radiotherapy compared with cisplatin chemo-radiotherapy in patients with unresectable squamous cell cancer of the esophagus. Radiother Oncol. 2007:83(2):139-47.

28. Chen Y, Ye J, Zhu Z, Zhao W, Zhou J, Wu C, Tang H, Fan M, Li L, Lin Q, et al. Comparing paclitaxel plus fluorouracil versus Cisplatin plus fluorouracil in Chemoradiotherapy for locally advanced esophageal squamous cell Cancer: a randomized, multicenter, phase III clinical trial. J Clin Oncol. 2019:37(20):1695-703.

29. Smith TJ, Ryan LM, Douglass HO Jr, Haller DG, Dayal Y, Kirkwood J, Tormey DC, Schutt AJ, Hinson J, Sischy B. Combined chemoradiotherapy vs. radiotherapy alone for early stage squamous cell carcinoma of the esophagus: a study of the eastern cooperative oncology group. Int J Radiat Oncol Biol Phys. 1998;42(2):269-76.

30. Yamashita H, Okuma K, Wakui R, Kobayashi-Shibata S, Ohtomo K, Nakagawa K. Details of recurrence sites after elective nodal irradiation (ENI) using 3Dconformal radiotherapy (3D-CRT) combined with chemotherapy for thoracic esophageal squamous cell carcinoma - a retrospective analysis. Radiother Oncol. 2011;98(2):255-60.

31. Ding X, Zhang J, Li B, Wang Z, Huang W, Zhou T, Wei Y, Li H. A metaanalysis of lymph node metastasis rate for patients with thoracic oesophageal cancer and its implication in delineation of clinical target volume for radiation therapy. Brit J Radiol. 2012;85(1019):E1110-9.

32. Yu WW, Zhu ZF, Fu XL, Zhao KL, Mao JF, Wu KL, Yang HJ, Fan M, Zhao S, Welsh J. Simultaneous integrated boost intensity-modulated radiotherapy in esophageal carcinoma early results of a phase II study. Strahlenther Onkol. 2014;190(11):979-86

33. Welsh J, Settle SH, Amini A, Xiao L, Suzuki A, Hayashi Y, Hofstetter W, Komaki R, Liao Z, Ajani JA. Failure patterns in patients with esophageal cancer treated with definitive chemoradiation. Cancer. 2012;118(10):2632-40.

34. Song T, Liang XD, Fang M, Wu SX. High-dose versus conventional-dose irradiation in cisplatin-based definitive concurrent chemoradiotherapy for esophageal cancer: a systematic review and pooled analysis. Expert Rev Anticanc. 2015;15(10):1157-69.

35. Li C, Ni WJ, Wang X, Zhou ZM, Deng W, Chang X, Chen DF, Feng QF, Liang J, Wang $X Z$, et al. A phase $|/|$ radiation dose escalation trial using simultaneous integrated boost technique with elective nodal irradiation and concurrent chemotherapy for unresectable esophageal Cancer. Radiat Oncol. 2019;14:48.

36. Chen D, Menon H, Verma V, Seyedin SN, Ajani JA, Hofstetter WL, Nguyen QN, Chang JY, Gomez DR, Amini A, et al. Results of a phase 1/2 trial of Chemoradiotherapy with simultaneous integrated boost of radiotherapy dose in Unresectable locally advanced esophageal Cancer. JAMA Oncol. 2019;5(11):1597-604
37. Yu W, Cai X-W, Liu Q, Zhu Z-F, Feng W, Zhang Q, Zhang Y-J, Yao Z-F, Fu X$\mathrm{L}$. Safety of dose escalation by simultaneous integrated boosting radiation dose within the primary tumor guided by 18FDG-PET/CT for esophageal cancer. Radiother Oncol. 2015;114(2):195-200.

38. Welsh J, Palmer MB, Ajani JA, Liao Z, Swisher SG, Hofstetter WL, Allen PK, Settle SH, Gomez D, Likhacheva A, et al. Esophageal Cancer Dose Escalation Using a Simultaneous Integrated Boost Technique. Int J Radiat Oncol Biol Phys. 2012;82(1):468-74.

39. Yang H, Liu H, Chen Y, Zhu C, Fang W, Yu Z, Mao W, Xiang J, Han Y, Chen $Z$, et al. Neoadjuvant Chemoradiotherapy followed by surgery versus surgery alone for locally advanced squamous cell carcinoma of the esophagus (NEOCRTEC5010): a phase III multicenter, randomized, OpenLabel Clinical Trial. J Clin Oncol. 2018;36(27):2796-803.

40. van Hagen P, Hulshof MC, van Lanschot JJ, Steyerberg EW, van Berge Henegouwen MI, Wijnhoven BP, Richel DJ, Nieuwenhuijzen GA, Hospers GA Bonenkamp JJ, et al. Preoperative chemoradiotherapy for esophageal or junctional cancer. N Engl J Med. 2012;366(22):2074-84.

41. Tong DK, Law S, Kwong DL, Chan KW, Lam AK, Wong KH. Histological regression of squamous esophageal carcinoma assessed by percentage of residual viable cells after neoadjuvant chemoradiation is an important prognostic factor. Ann Surg Oncol. 2010;17(8):2184-92.

42. Meguid RA, Hooker CM, Taylor JT, Kleinberg LR, Cattaneo SM 2nd, Sussman MS, Yang SC, Heitmiller RF, Forastiere AA, Brock MV. Recurrence after neoadjuvant chemoradiation and surgery for esophageal cancer: does the pattern of recurrence differ for patients with complete response and those with partial or no response? J Thorac Cardiovasc Surg. 2009;138(6):1309-17.

43. Kim MK, Kim SB, Ahn JH, Kim YH, Kim JH, Jung HY, Lee GH, Choi KD, Song $\mathrm{HY}$, Shin $\mathrm{JH}$, et al. Treatment outcome and recursive partitioning analysisbased prognostic factors in patients with esophageal squamous cell carcinoma receiving preoperative chemoradiotherapy. Int J Radiat Oncol Biol Phys. 2008;71(3):725-34.

44. He L, Allen PK, Potter A, Wang J, Chang JY, Gomez DR, Komaki R, Liao Z, Lin $\mathrm{SH}$. Re-evaluating the optimal radiation dose for definitive chemoradiotherapy for esophageal squamous cell carcinoma. J Thorac Oncol. 2014;9(9):1398-405.

45. van Hagen P, van Lanschot JJB, van der Gaast A. Preoperative Chemoradiotherapy for esophageal Cancer reply. New Engl J Med. 2012; 367(9):873-4.

\section{Publisher's Note}

Springer Nature remains neutral with regard to jurisdictional claims in published maps and institutional affiliations.
Ready to submit your research? Choose BMC and benefit from:
- fast, convenient online submission
- thorough peer review by experienced researchers in your field
- rapid publication on acceptance
- support for research data, including large and complex data types
- gold Open Access which fosters wider collaboration and increased citations
- maximum visibility for your research: over $100 \mathrm{M}$ website views per year
At BMC, research is always in progress.
Learn more biomedcentral.com/submissions 